\title{
Cancer Treatment During COVID-19: Resilience of Individuals With Advanced Non-Small Cell Lung Cancer Versus Community Controls
}

Nicole A. Arrato, MA ${ }^{1}$; Stephen B. Lo, MA ${ }^{1}$; Clarence A. Coker, BSc ${ }^{1}$; Jonathan J. Covarrubias, MS ${ }^{1}$; Tessa R. Blevins, BA ${ }^{1}$; Sarah A. Reisinger, PhD, MPH, MCHES²; Carolyn J. Presley, MD, MHS ${ }^{3}$; Peter G. Shields, $\mathrm{MD}^{2,3}$; and

Barbara L. Andersen, $\mathrm{PhD}^{1}$

\section{ABSTRACT}

Background: Among all patients with cancer, those with advanced non-small cell lung cancer (NSCLC) experience the most distress. Although new therapies are improving survival, it is unknown whether receiving immunotherapy or targeted therapy during the COVID-19 pandemic increases patients' psychological vulnerability. To meet clinical needs, knowledge of patients' COVID-19 perceptions and safety behaviors is essential. Thus, this study compared patients' psychological responses at diagnosis and during COVID-19 and compared patients with similar individuals without cancer during the same period. Patients and Methods: Patients with advanced NSCLC enrolled at diagnosis for cohort study participated (ClinicalTrials.gov identifier: NCT03199651). Those with follow-ups from April 28, 2020, through July 14, $2020(n=76)$, were assessed again including COVID19 measures. Simultaneously, community controls with similar sociodemographics and smoking histories were solicited $(n=67)$. Measures were COVID-19 perceptions (Brief Illness Perception Questionnaire), social distancing, and depressive (Patient Health Questionnaire-9) and anxiety (Generalized Anxiety Disorder-7) symptoms. First, analyses evaluated differences in the psychological responses of patients with NSCLC at diagnosis and during COVID-19. Second, patients and controls were contrasted on COVID-19 perceptions, social distancing, and psychological symptoms. Results: The depressive and anxious symptoms of patients with NSCLC were greater at diagnosis $(P<.02)$ than during COVID-19, approximately 1 year later. Patients with NSCLC and controls did not differ in terms of sociodemographics, except those with NSCLC were more racially diverse and older, and had greater smoking history $(P<.03)$. Groups did not differ regarding concern, understanding, or perceived control over COVID-19 ( $P>.406)$. Notably, controls anticipated the COVID threat would last longer, practiced more social distancing, were more concerned about family $(P<.04)$, and reported worse psychological symptoms $(P<.023)$. With less depression and anxiety, patients with NSCLC viewed COVID-19 as a shorter-term threat and had fewer COVID-19-related worries than did controls. For controls, COVID19 was more salient, heightening worries and psychological symptoms. Conclusions: Despite multiple health stressors, patients with NSCLC demonstrated resilience when receiving cancer treatment during COVID-19. Nonetheless, this population remains psychologically vulnerable, requiring support at diagnosis and thereafter.

J Natl Compr Canc Netw 2022;20(2):118-125 doi: 10.6004/jnccn.2021.7076

${ }^{1}$ Department of Psychology, ${ }^{2}$ Comprehensive Cancer Center, and ${ }^{3}$ Division of Medical Oncology, Department of Internal Medicine, College of Medicine, The Ohio State University, Columbus, Ohio.

\section{Background}

Patients with cancer are more vulnerable to COVID-19 than otherwise healthy individuals. Patients with lung cancer (LC) are uniquely vulnerable to coronaviral pneumonias (severe acute respiratory syndrome coronavirus 2 [SARS-CoV-2]), along with acute respiratory distress syndrome, and if infected, have a greater likelihood of dying. ${ }^{1,2}$ COVID-19 notwithstanding, the scope of the LC problem is without parallel. ${ }^{3}$ In the United States, LC accounts for $25 \%$ more cancer deaths than deaths from prostate, breast, and colon cancers combined, ${ }^{4}$ partly because $84 \%$ of patients with LC are diagnosed at an advanced stage. ${ }^{5}$ Non-small cell LC (NSCLC) accounts for $85 \%$ of all LCs and is the fifth costliest tumor to treat, with estimated national expenditures of $\$ 14.7$ billion for $2020 .^{6}$

As the pandemic continues, patients with LC are, unfortunately, vulnerable for other reasons: they are the most emotionally distressed of all cancer groups, ${ }^{7}$ having the greatest prevalence of mood and anxiety disorders. ${ }^{8}$ At diagnosis, many patients with advanced (stage IV) NSCLC perceive cancer as consequential and uncontrollable, even in the context of new immunotherapy and targeted treatments. ${ }^{9}$ Approximately $40 \%$ report moderate to severe depressive symptoms, anxiety, and trauma levels of cancer-specific stress, ${ }^{10}$ and cross-sectional studies postdiagnosis show heightened symptom levels. ${ }^{11,12}$ Many might assume that COVID-19 adds further stress to patients with NSCLC, although the extent to which this occurs is unknown. It is possible, however, that this population actually exhibits a positive adaptation known as resilience, which is the ability to maintain or regain mental health despite experiencing adversity. ${ }^{13}$ Without information, providers may be ill-prepared to meet patients' needs during this difficult time and may

See JNCCN.org for supplemental online content. 
not know the extent to which COVID-19 exacerbates patients' stress, particularly as they try to continue therapy during the pandemic. Such issues are time-sensitive, considering the devastation attributable to mental health consequences of the pandemic, ${ }^{14}$ the multiple waves of COVID-19 cases in the United States, and the stalling of vaccine uptake. ${ }^{15}$

Patients with NSCLC, enrolled at diagnosis and followed for a cohort study (ClinicalTrials.gov identifier: NCT03199651), enabled analysis of 2 important issues. First, patients' psychological responses at diagnosis (pre-COVID-19) were compared with those during COVID-19. Prior to COVID-19, many cancer groups have shown a declining trajectory of stress or depression from diagnosis across time. ${ }^{16-18}$ We were uncertain if that trend would still be evidenced with patients in treatment amid the pandemic. Second, the responses of patients with NSCLC during COVID-19 were compared with a time-equivalent normative sample, namely, similar individuals from the community also coping with COVID-19 but cancer-free. Having health risk from multiple sources, would patients with NSCLC show greater negative affect, such as pessimism or worry, or difficulties with social distancing than others trying to cope while free of the added cancer burden? Relevant to clinical care, would the picture for patients with LC be one of unique, added burden or one, possibly, of resilience?

The study was performed in Ohio as infections were beginning to increase in April 2020. At that time, 750 new cases were occurring per day (21,000 total), a rate that remained stable until early June 2020 . Thereafter, cases accelerated at more than double the daily rate $(n=1,630)$ and approached a total nearly 4 times higher $(n=78,000)$ by the end of data collection (July 2020). Patients with NSCLC still enrolled and alive completed a COVID-19-specific assessment during the study window; archival psychological data from the patients at diagnosis were also used. As COVID-19 risk varies across time and US states, data from comparison individuals (controls) residing in Ohio were obtained during the same window.

\section{Patients and Methods}

Procedures

The Institutional Review Board of The Ohio State University approved the study and all procedures in accordance with the Declaration of Helsinki. All participants completed written informed consent. A group comparison design was used.

The NCI-designated Ohio State University Comprehensive Cancer Center enrolled patients with stage IV NSCLC at diagnosis into an observational cohort (Beating Lung Cancer in Ohio [BLCIO]; ClinicalTrials.gov identifier: NCT03199651) beginning in 2017. Supplemental eTable 1 provides inclusion/exclusion criteria (available with this article at JNCCN.org). Patient-reported outcomes for anxiety and depressive symptoms were completed by telephone at diagnosis with the aid of a survey research firm. In the same manner, patient follow-ups scheduled from April 28, 2020, to July 14, 2020, were performed with COVID-19-specific items additionally completed. Of the 99 patients to be assessed in the time window, 76 were completed (76.8\%). Reasons for the 23 missing responses were: 4 too ill to complete assessment; 9 contacted but not completed before assessment window (30 days) closed; and 10 not contacted within assessment window. Overall, BLCIO has a mean completion rate of $80 \%$ of available patients (those still alive) across follow-ups from diagnosis to 24 months.

Published data from the cohort at diagnosis $(n=186$ through April 2019) showed a mean age of 62.5 years, $91.4 \%$ with a smoking history, low education and income levels, and all patients residing in Ohio. ${ }^{10}$ These characteristics guided the solicitation of controls. Because difficulty with matching was anticipated, 2 web-based platforms (ResearchMatch, CloudResearch) were used with the following participation criteria: no prior or current cancer diagnosis, age 45-90 years, high school education or less, $\leq \$ 75,000$ annual income, current or former smoker, Ohio resident, and ability to complete online assessments in English. From May 8, 2020, to June 19, 2020, 67 control individuals enrolled and completed measures on Qualtrics.

\section{Measures}

\section{Psychological Symptoms}

ASCO-recommended measures were used. ${ }^{19}$ Symptoms of major depressive disorder were assessed using the Patient Health Questionnaire-9 (PHQ-9) ${ }^{20}$ measure. The PHQ-9 total score can range from 0 to 27, with each of 9 items measured on a Likert scale from 0 (not at all) to 3 (nearly every day). Symptoms of generalized anxiety disorder were assessed with the Generalized Anxiety Disorder-7 (GAD-7) ${ }^{21}$ measure. The GAD-7 total score can range from 0 to 21, with each of 7 items measured on the same Likert scale as the PHQ-9. Cutoffs suggestive of at least moderate symptom levels are $>9$ for both measures.

\section{General Health Concern}

Patients with NSCLC rated the following item: "Has the COVID-19 pandemic worsened your concern about your lung cancer?," and controls rated "Has the COVID-19 pandemic worsened your concern about your health?" The scale for each ranged from 0 (not at all concerned) to 10 (extremely concerned). 


\section{COVID-19 Measures}

\section{Illness Perceptions}

The Brief Illness Perception Questionnaire ${ }^{22}$ assessed patients' and controls' perceptions of COVID-19 (its consequences, timeline, personal control, concern, understanding). For example, the timeline item asked, "How long do you think the COVID-19 pandemic will continue?" The concern item asked, "How concerned are you about getting COVID-19?" Each item was rated from 0 to 10 . Two items that were not relevant to COVID-19 were omitted (treatment control, identity/symptoms) (see supplemental eTable 2 for complete description).

\section{Exposure, Social Distancing, and Stress/Worry}

For infection tally, items from Wang et $\mathrm{al}^{23}$ were used. Three items assessed knowledge (number) of persons with COVID-19 in the family, friends, and acquaintances (eg, "How many family members have been diagnosed with COVID-19?"). For social distancing, one item was used, which was rated from 0 (not at all) to 6 (all the time): "Have you been able to 'socially distance' yourself from household members?" For stress and worry, 3 items were used. The questions "How would you rate your stress during isolation or social distancing?" and "Has isolation or social distancing created tension in your household?" were both rated on a 0 - to 6-point scale, with higher scores indicating more stress/tension, and "How worried are you that any of your close family members will contract COVID-19?" was rated from 0 (not worried at all) to 10 (extremely worried) (see supplemental eTable 2 for complete description).

\section{Descriptive}

Sociodemographic items were included. For patients with NSCLC, data were obtained from the electronic medical record and verified by 2 raters. NSCLC disease and treatment characteristics were obtained as well as the Charlson comorbidity index (CCI ${ }^{24}$ quantitatively summarizing the presence and severity of 17 common medical conditions. CCI scores ranged from 0 to 37 ; NSCLC scores were a minimum of 6 for "metastatic solid tumor.” Controls self-rated all CCI items.

\section{Statistical Analysis}

One-way ANOVAs and chi-square tests examined group differences in demographics. A Fisher exact test was run for race due to low variability $(n=3$ for non-White participants in the control group). Variables significantly different between groups were used as controls.

For the first analyses, paired sample $t$ tests were used to test change in the depressive and anxiety symptoms from diagnosis (pre-COVID-19) to followup during COVID-19 of patients with NSCLC. Second, multivariate linear regressions tested for group differences in outcomes, controlling for variables significantly different by group. A $P$ value $<.05$ was considered statistically significant. A sensitivity analysis using the gpower program ${ }^{25}$ was performed to confirm a sufficient sample size. Sensitivity analysis indicated a small-medium effect size of group differences in outcomes $\left(f^{2}=0.106\right)$.

\section{Results \\ Description}

\section{Patients With NSCLC}

Comparing our sample to that of the SEER program, ${ }^{26}$ the NSCLC group was younger (mean, 62.6 years; range, 29-80 years), roughly equivalent in terms of sex (female, $\mathrm{n}=31 ; 40.8 \%$ ), married/partnered $(65.8 \%)$, and less ethnically/racially diverse $(80.3 \%$ White) (Table 1). The sample was equivalent to SEER in that most of the tumors were adenocarcinoma (77.6\%). Other sample characteristics included education level less than high school for $6.6 \%(n=5)$, high school for $35.5 \%(\mathrm{n}=27)$, more than high school for $57.9 \%$ $(n=44)$, and income, with $21.1 \%(n=16)$ below the Ohio poverty line for a family of $4(\$ 25,000)$. At followup, all patients except 5 were in treatment with novel immunotherapies or targeted therapies.

\section{Controls: Comparison of Groups on Sociodemographics} Similarity of samples was approximated. NSCLC and control groups did not differ in terms of sex, education, income, marital status, or residence (Ohio). The control group was less diverse ( $5 \%$ vs $20 \%$ non-White; odds ratio [OR], 5.246; 95\% CI, 1.447-19.024; $P=.006$ ), younger (59 vs 63 years; Cohen $d=0.392 ; P=.023$ ), and had somewhat fewer current/former smokers $(75 \%$ vs 90\%; OR, 2.890; 95\% CI, 1.156-7.225; $P=.020$ ) (Table 1). Thus, the latter variables were entered as controls in the multivariate linear regression analyses.

\section{Psychological Responses of Patients With NSCLC at Diagnosis Versus During COVID-19}

Patients with NSCLC reported significantly more depressive $(\Delta \mathrm{M}=1.737 ; 95 \% \mathrm{CI}, 0.493-2.98 ; P=.007)$ and anxiety symptoms $(\Delta \mathrm{M}=1.592 ; 95 \% \mathrm{CI}, 0.264-2.920 ; P=.019)$ at diagnosis (pre-COVID-19) than during COVID-19. Percentages of patients with NSCLC above cutoff values at diagnosis and at COVID-19 follow-up were $17.1 \%(n=13)$ versus $11.8 \%(n=9)$ on the PHQ-9 and $15.8 \%(n=12)$ versus $13.2 \%(n=10)$ on the GAD-7, respectively. 


\begin{tabular}{|c|c|c|c|}
\hline Variable & $\begin{array}{c}\text { Patients } \\
\text { With NSCLC } \\
\text { n (\%) }\end{array}$ & $\begin{array}{l}\text { Controls } \\
\text { n (\%) }\end{array}$ & $P$ Value \\
\hline Total, $n$ & 76 & 67 & \\
\hline Age, mean $[S D]$ & $62.6[11.08]$ & $58.6[9.31]$ & .023 \\
\hline Race & & & .006 \\
\hline White & $61(80.3)$ & $64(95.5)$ & \\
\hline Non-White & $15(19.7)$ & $3(4.5)$ & \\
\hline Sex & & & .058 \\
\hline Male & $45(59.2)$ & $29(43.3)$ & \\
\hline Female & $31(40.8)$ & $38(56.7)$ & \\
\hline Marital status & & & .329 \\
\hline Currently married/partnered & $50(65.8)$ & $36(53.7)$ & \\
\hline Single, never married & $8(10.5)$ & $9(13.4)$ & \\
\hline Separated or divorced & $12(15.8)$ & $20(29.9)$ & \\
\hline Widowed & $6(7.9)$ & $2(3.0)$ & \\
\hline Education & & & .285 \\
\hline $\begin{array}{l}<8 \text { th grade or some high } \\
\text { school }\end{array}$ & $5(6.6)$ & $4(6.0)$ & \\
\hline High school diploma/GED & $27(35.5)$ & $19(28.4)$ & \\
\hline $\begin{array}{l}\text { Some college/undergraduate } \\
\text { degree }\end{array}$ & $33(43.4)$ & $30(44.8)$ & \\
\hline Graduate degree & $11(14.5)$ & $14(20.9)$ & \\
\hline Income & & & .352 \\
\hline$\leq \$ 15,000$ & $6(7.9)$ & $8(11.9)$ & \\
\hline$\$ 15,001-\$ 25,000$ & $10(13.2)$ & $3(4.5)$ & \\
\hline$\$ 25,001-\$ 50,000$ & $21(27.6)$ & $12(17.9)$ & \\
\hline$\$ 50,001-\$ 75,000$ & $10(13.2)$ & $15(22.4)$ & \\
\hline$\$ 75,001-\$ 100,000$ & $8(10.5)$ & $17(25.4)$ & \\
\hline$\geq \$ 100,001$ & $16(21.1)$ & $12(17.9)$ & \\
\hline Do not know/missing & $5(6.6)$ & - & \\
\hline Smoking (\% ever) & $68(89.5)$ & $50(74.7)$ & .020 \\
\hline $\mathrm{CCl}$ score, mean $[\mathrm{SD}]$ & 9.40 [1.93] & $2.46[2.00]$ & $<.001$ \\
\hline $\begin{array}{l}\text { Months since diagnosis } \\
\text { to COVID-19 assessment, } \\
\text { mean [SD] }\end{array}$ & $12.4[6.9]^{\mathrm{a}}$ & - & \\
\hline \multicolumn{4}{|l|}{ Cell type } \\
\hline Adenocarcinoma & $59(77.6)$ & - & \\
\hline Squamous & $12(15.8)$ & - & \\
\hline Other & $4(5.3)$ & - & \\
\hline Missing & $1(1.3)$ & - & \\
\hline \multicolumn{4}{|l|}{ Treatment type } \\
\hline Immunotherapy & $12(15.8)$ & - & \\
\hline $\begin{array}{l}\text { Chemotherapy }+ \\
\text { immunotherapy }\end{array}$ & $33(43.4)$ & - & \\
\hline Targeted therapy & $20(26.3)$ & - & \\
\hline Chemotherapy & $5(6.6)$ & - & \\
\hline Not available & $6(7.9)$ & - & \\
\hline
\end{tabular}

Abbreviation: NSCLC, non-small cell lung cancer.

a Median, 10.7 months (range, 3.0-34.2 months).
Multivariate Linear Regressions Comparing Patients With NSCLC and Controls During COVID-19

\section{Psychological Symptoms}

Notably, patients with NSCLC reported significantly fewer depressive $(\beta=-0.206 ; 95 \% \mathrm{CI},-4.142$ to $-0.355 ; P=.020)$ and anxiety symptoms $(\beta=-0.196$; $95 \%$ CI, -4.037 to $-0.308 ; P=.023$ ) than controls, with racial group, age, and smoking status controlled (Table 2 ). Percentages of individuals above cutoff values in the NSCLC and control groups were $11.8 \%$ versus $28.1 \%$ on the PHQ- 9 and $13.2 \%$ versus $30.3 \%$ on the GAD-7, respectively.

\section{Health Concern}

COVID-19 worsened controls' moderate concern about their health (mean score, 5.45), and interestingly, this was similar $(P=.943)$ to the moderately worsening concern about cancer among patients with NSCLC (mean score, 5.91; Table 2).

\section{COVID-19 Analyses}

\section{Illness Perceptions}

Controlling for racial group, age, and smoking status, patients with NSCLC and controls perceived COVID-19 similarly. Both perceived they had high knowledge about COVID-19, felt they had similar (moderate) control over their own COVID-19 risk, and had similar levels of concern about contracting COVID-19. However, unlike patients with NSCLC, controls anticipated the COVID-19 threat would last significantly longer $(\beta=-0.278$; $95 \%$ CI, -0.925 to $-0.180 ; P=.004$ ).

\section{Exposure, Social Distancing, and Stress/Worry}

Controlling for relevant variables, groups did not differ on knowledge of persons with COVID-19. However, patients with NSCLC reported being significantly more successful with social distancing $(\beta=0.300 ; 95 \% \mathrm{CI}$, $0.217-0.979 ; \quad P=.002)$, less stressed about COVID-19 ( $\beta=-0.177 ; 95 \% \mathrm{CI},-0.701$ to $-0.002 ; P=.049$ ), and less worried about family contracting COVID-19 ( $\beta=-0.192$; $95 \%$ CI, -0.734 to $-0.028 ; P=.035)$ compared with controls.

\section{Discussion}

To our knowledge, this is the first empirical, controlled comparison study of the psychological and behavioral impacts of COVID-19 among US patients with cancer. The data are novel, because thus far, such studies have come from Europe and Asia only. ${ }^{27,28}$ Patients with LC are an important group, representing nearly $13 \%$ of all new cancer cases in the United States annually. Survival for those with advanced NSCLC is changing dramatically as new immunotherapies and targeted therapies become 
available and treatment guidelines shift regularly. Thus, it is important to understand and appreciate how patients with NSCLC with the most severe disease burden and symptom severity-ones uniquely susceptible to COVID19 infection and death-are emotionally and behaviorally able to travel to the hospital and receive treatment, monthly if not weekly. Results are discussed in terms of the resilience of patients with NSCLC and the differential salience $^{29}$ of COVID-19 for the controls. Implications for clinical practice are provided.

As noted, ${ }^{7}$ patients with LC are regularly identified with the highest levels of stress or depression, and depression at diagnosis predicts premature death for them (eg, hazard ratio, 1.39; 95\% CI, $1.24-1.56) .{ }^{30}$ PreCOVID-19, a downward trajectory of psychological symptom severity from diagnosis onward has been found for patients with cancer. ${ }^{16-18}$ Some might view the significant declines in the psychological symptoms of patients with NSCLC from diagnosis to follow-up as a response shift. ${ }^{31}$ In response shift, patients recalibrate the meaning of questionnaire items, particularly if items are general, such as "rate your quality of life." Response shift is unlikely for the PHQ-9/GAD-7 items, as they are the $D S M-I V$ criteria for major depressive disorder and generalized anxiety disorder, and thus by design are not open to interpretation. The items have rigorous reliability and validity support. Therefore, we view the lower levels of depressive and anxiety symptoms of patients with NSCLC during COVID-19 as accurately reflecting their self-reported psychological status.

It is notable how low the patients' PHQ-9/GAD-7 scores were during the COVID-19 pandemic. When designing the study, some expected the symptom scores to be elevated. To the contrary, the data characterize the patients as resilient in the face of COVID-19. Resiliency is adapting well in the face of adversity, trauma, tragedy, threats, or significant stress (colloquially, "being tough"). ${ }^{13,32}$ As defined, resiliency combines adversity or risk with positive outcomes, with the positive outcome often equated to a person "retaining their mental health and not succumbing to a mental illness." ${ }^{33}$ Evidence for the resilience of patients with NSCLC includes reports of

Table 2. Multivariate Regression Analyses of Depressive and Anxiety Symptoms, Perceptions, Exposure, Social Distancing, and Worries

\begin{tabular}{|c|c|c|c|c|c|c|c|}
\hline Measure & \multicolumn{3}{|c|}{ Patients With NSCLC } & \multicolumn{3}{|c|}{ Controls } & P Value ${ }^{a}$ \\
\hline \multicolumn{8}{|l|}{ Psychological symptoms } \\
\hline GAD-7c & $3.67[5.12]$ & $0-21$ & - & $6.30[5.63]$ & $0-20$ & 1 & .023 \\
\hline Concern re: cancer/health & $5.91[3.63]$ & $0-10$ & - & $5.45[3.22]$ & $0-10$ & - & .943 \\
\hline Consequences & $5.92[3.26]$ & $0-10$ & - & $6.04[2.36]$ & $0-10$ & - & .878 \\
\hline Anticipated timeline & $5.83[2.12]$ & $0-10$ & 4 & $6.78[1.65]$ & $4-10$ & 8 & .004 \\
\hline Personal control & $6.40[2.84]$ & $0-10$ & 1 & $5.78[2.09]$ & 0-10 & 3 & .474 \\
\hline Comprehension & $8.29[2.25]$ & $0-10$ & 1 & $8.51[1.80]$ & $2-10$ & - & .406 \\
\hline Concern & $6.01[3.57]$ & $0-10$ & 1 & $6.07[2.91]$ & $0-10$ & - & .409 \\
\hline Acquaintance & $0.16[0.75]$ & $0-6$ & - & $0.42[1.40]$ & $0-10$ & 4 & .406 \\
\hline \multicolumn{8}{|l|}{ Social distancing } \\
\hline Within household & $3.13[2.81]$ & $0-6$ & 14 & $1.54[2.28]$ & $0-6$ & 10 & .002 \\
\hline \multicolumn{8}{|l|}{ Stress/Worry } \\
\hline Personal stress & $1.82[1.81]$ & $0-6$ & - & $2.64[1.87]$ & $0-6$ & - & .049 \\
\hline Household stress & $1.16[1.82]$ & $0-6$ & 13 & $1.41[1.76]$ & $0-6$ & 11 & .403 \\
\hline Worry re: family & $5.41[3.40]$ & $0-10$ & - & $6.29[1.54]$ & 0-10 & 2 & .035 \\
\hline
\end{tabular}

Abbreviations: GAD-7, Generalized Anxiety Disorder-7; NSCLC, non-small cell lung cancer; PHQ-9, Patient Health Questionnaire-9.

${ }^{a}$ Age, race, and smoking status were included as control variables.

${ }^{\mathrm{b}} \mathrm{A}$ higher PHQ-9 score indicates greater depressive symptoms.

${ }^{\mathrm{C}} \mathrm{A}$ higher GAD-7 score indicates greater anxiety symptoms. 


\section{Table 3. Implications for Clinical Practice}

Comparison of stressors. Novel data show symptoms of depression and anxiety are higher at NSCLC diagnosis than during COVID-19, approximately 1 year later.

Recognize resilience. Despite dual threats, patients with NSCLC were resilient, reporting high levels of COVID-19-related understanding and keeping COVID-19 worries to a moderate level, no higher than that of healthy controls.

Psychological challenges. Patients with advanced NSCLC remain the most psychologically challenged of all patients with cancer, with significant needs for vigorous supportive care to achieve survival benefits of new therapies during the pandemic.

Depression and anxiety screening during the pandemic. ASCO-recommended, validated measures for depression (PHQ-9) and anxiety (GAD-7) should be used at diagnosis and periodically thereafter.

Screening necessitates referral. Patients with positive psychological screens require further evaluation and possible referral for empirically supported, psychological treatment.

Abbreviations: GAD-7, Generalized Anxiety Disorder-7; NSCLC, non-small cell lung cancer; PHQ-9, Patient Heath Questionnaire-9.

significantly less stress, less worry about their family contracting the virus, greater success with social distancing, and having no/few psychological symptoms at the time. This does not mean the patients with NSCLC were unfazed by the COVID-19 pandemic, because they had moderate concern about their lung cancer, but the latter was of similar magnitude to the controls' concern about their health. These data contextualize the picture of how these patients with cancer are responding during the pandemic. That is, patients with NSCLC had cancer plus high COVID-19 risk and its associated comorbidities (eg, heart disease, chronic obstructive pulmonary disease), and the stigma of having a cancer that others view as self-imposed. ${ }^{34}$

Group differences may be also understood by differential salience of COVID-19. US studies have shown heightened COVID-19 anxiety and fear in the general population. ${ }^{35,36}$ For otherwise healthy controls without chronic conditions (CCI score $=2$ ) and life routines unchanged, COVID-19 was a salient (ie, novel, unexpected) stressor. Compared with patients with NSCLC, controls reported significantly more stress, worry, and higher psychological symptoms, with more than twice the percentage of individuals scoring above diagnostic cutoffs for anxiety (13\%) and depression (28\%). ${ }^{37}$ Evidence for differential salience is, perhaps, most obvious in that controls were as concerned about their health as were individuals with advanced NSCLC. By contrast, COVID-19 for patients with NSCLC occurred in the midst of ongoing life threat, comorbidities, symptoms, and routines already disrupted by receiving cancer treatment.

Implications for clinical practice are considered (Table 3). Because data show that symptoms of depression and anxiety are higher at NSCLC diagnosis than thereafter, it is suggested that providers continually consider each patient's timeline, especially during the COVID-19 pandemic. Providers may recognize resilience and also consider that patients with NSCLC continue to require supportive care to achieve survival benefits of new therapies. ASCO-recommended measures for depression and anxiety should be used at diagnosis and thereafter, and patients with positive screens would likely benefit from psychological treatment.

Research design and methodological aspects of the study are considered. This study addresses a gap, not only of psychological studies of patients with NSCLC receiving new therapies but also of patients with cancer during the COVID-19 pandemic. With any cross-sectional study of patients with cancer, it is relevant to appreciate the selection factor; in other words, the patients with NSCLC assessed during COVID-19 were those surviving "long enough" to reach an April 2020 follow-up, irrespective of time since diagnosis. However, the median time since diagnosis in this sample ( $\sim 1$ year) highlights the improving prognosis being achieved with new therapies, a sharp contrast to the 4-month median observed for decades. ${ }^{38}$ Equivalence of geographic region and timing are key factors in choosing control conditions for studies of COVID-19. In general, it is difficult to obtain online research study volunteers for comparison with individuals who are older, have lower education/income, and have negative health behaviors such as smoking. We were fortunate that the strategy for control accrual resulted in no statistical differences in several social determinants of health, including marital/partner status, education, and income. Notably, the 2019 US Census poverty rate in Ohio was $13.1 \%$, but $20 \%$ of individuals in both study groups live below the US-defined poverty line. Still, broad generalization of the control findings to similar others who are not research platform users is unknown.

\section{Conclusions}

New data provide perspective for appreciating the COVID19 experience for patients with cancer at greatest risk. Patients with cancer may be more resilient to COVID-19 stressors than has been assumed or suggested. ${ }^{37}$ Although not immune to the stresses of COVID-19, data show resilience for otherwise heavily burdened patients with NSCLC - ones unable to "shelter in place" but leaving home regularly to receive treatment. Although recent data from $>260,000$ cancer survivors show patients with LC 
experience greater distress and disability and poorer quality of life than those with other common cancers, ${ }^{39}$ the latter studies do not necessarily capture the whole story. The present data show that, in the face of multiple health stressors and COVID-19, patients with NSCLC demonstrated resilience.

\section{Acknowledgments}

The authors wish to acknowledge the study participants for making this research possible and Dr. Samilia Obeng-Gyasi for comments on the manuscript. The authors acknowledge the Strategic Research Group, ResearchMatch, and CloudResearch for their contributions to data collection.
Submitted February 22, 2021; final revision received June 7, 2021; accepted for publication June 18, 2021.

Author contributions: Study concept: Arrato, Andersen. Investigation: Arrato, Andersen. Data curation: Arrato, Coker, Covarrubias, Reisinger, Presley, Andersen. Formal analysis: Lo, Coker, Covarrubias. Funding acquisition: Shields. Methodology: Lo, Andersen. Supervision: Reisinger, Presley, Shields. Validation: Covarrubias. Writing - original draft: Arrato, Lo, Coker, Blevins, Andersen. Writing - review and editing: Arrato, Lo, Coker, Blevins, Reisinger, Presley, Shields, Andersen.

Disclosures: The authors have disclosed that they have not received any financial considerations from any person or organization to support the preparation, analysis, results, or discussion of this article.

Funding: This work was supported by funding from Ohio State University Comprehensive Cancer Center Pelotonia.

Correspondence: Barbara L. Andersen, PhD, Department of Psychology, The Ohio State University, 1835 Neil Avenue, Columbus, OH 43210-1222. Email: Andersen.1@osu.edu

\section{References}

1. Rogado J, Pangua C, Serrano-Montero G, et al. Covid-19 and lung cancer: a greater fatality rate? Lung Cancer 2020;146:19-22.

2. Elkrief $A$, Kazandjian $S$, Bouganim N. Changes in lung cancer treatment as a result of the coronavirus disease 2019 pandemic. JAMA Oncol 2020;6:1805-1806.

3. Bray F, Ferlay J, Soerjomataram I, et al. Global cancer statistics 2018 : GLOBOCAN estimates of incidence and mortality worldwide for 36 cancers in 185 countries. CA Cancer J Clin 2018;68:394-424.

4. Guadagno A. World Lung Cancer Day 2019: facts \& figures. Accessed October 14, 2021. Available at: https://www.curetoday.com/view/worldlung-cancer-day-2019-facts-figures

5. Wong MCS, Lao XQ, Ho KF, et al. Incidence and mortality of lung cancer: global trends and association with socioeconomic status. Sci Rep 2017;7:14300

6. Mariotto $A B$, Yabroff $K R$, Shao $Y$, et al. Projections of the cost of cancer care in the United States: 2010-2020. J Natl Cancer Inst 2011;103:117-128.

7. Carlson LE, Zelinski EL, Toivonen Kl, et al. Prevalence of psychosocial distress in cancer patients across 55 North American cancer centers. J Psychosoc Oncol 2019;37:5-21.

8. Linden W, Vodermaier A, Mackenzie R, et al. Anxiety and depression after cancer diagnosis: prevalence rates by cancer type, gender, and age. J Affect Disord 2012;141:343-351.

9. Lo SB, Shields PG, Carbone DP, et al. Advanced non-small cell lung cancer (NSCLC): patients' illness perceptions at diagnosis. J Cancer Rehabil 2019;2:24-29.

10. Andersen BL, Valentine TR, Lo SB, et al. Newly diagnosed patients with advanced non-small cell lung cancer: a clinical description of those with moderate to severe depressive symptoms. Lung Cancer 2020;145: 195-204.

11. Kurtz ME, Kurtz JC, Stommel M, et al. Predictors of depressive symptomatology of geriatric patients with lung cancer-a longitudinal analysis. Psychooncology 2002;11:12-22.

12. Sullivan $D R$, Forsberg CW, Ganzini L, et al. Depression symptom trends and health domains among lung cancer patients in the CanCORS study. Lung Cancer 2016;100:102-109.

13. Herrman $\mathrm{H}$, Stewart DE, Diaz-Granados $\mathrm{N}$, et al. What is resilience? Can J Psychiatry 2011;56:258-265.

14. Simon NM, Saxe GN, Marmar CR. Mental health disorders related to COVID-19-related deaths. JAMA 2020;324:1493-1494.

15. Wilson C. The U.S. is entering a new COVID-19 vaccination crisis. Accessed June 6, 2021. Available at: https://time.com/6046880/covid19-vaccine-slowdown/

16. Andersen BL, Goyal NG, Westbrook TD, et al. Trajectories of stress, depressive symptoms, and immunity in cancer survivors: diagnosis to 5 years. Clin Cancer Res 2017;23:52-61.

17. Neilson K, Pollard A, Boonzaier A, et al. A longitudinal study of distress (depression and anxiety) up to 18 months after radiotherapy for head and neck cancer. Psychooncology 2013;22:1843-1848.

18. Trudel-Fitzgerald $\mathrm{C}$, Savard J, Ivers $\mathrm{H}$. Which symptoms come first? Exploration of temporal relationships between cancer-related symptoms over an 18-month period. Ann Behav Med 2013;45:329-337.
19. Andersen BL, DeRubeis RJ, Berman BS, et al. Screening, assessment, and care of anxiety and depressive symptoms in adults with cancer: an American Society of Clinical Oncology guideline adaptation. J Clin Oncol 2014:32:1605-1619.

20. Kroenke K, Spitzer RL, Williams JB. The PHQ-9: validity of a brief depression severity measure. J Gen Intern Med 2001;16:606-613.

21. Spitzer RL, Kroenke K, Williams JB, et al. A brief measure for assessing generalized anxiety disorder: the GAD-7. Arch Intern Med 2006;166: 1092-1097.

22. Broadbent $\mathrm{E}$, Petrie KJ, Main J, et al. The brief illness perception questionnaire. J Psychosom Res 2006;60:631-637.

23. Wang $C$, Pan $R$, Wan $X$, et al. Immediate psychological responses and associated factors during the initial stage of the 2019 coronavirus disease (COVID-19) epidemic among the general population in China. Int J Environ Res Public Health 2020;17:1729.

24. Charlson ME, Pompei P, Ales KL, et al. A new method of classifying prognostic comorbidity in longitudinal studies: development and validation. J Chronic Dis 1987;40:373-383.

25. Erdfelder E, Faul F, Buchner A. GPOWER: a general power analysis program. Behav Res Methods Instrum Comput 1996;28:1-11.

26. Surveillance, Epidemiology, and End Results Program. SEER*Stat Database. Accessed November 26, 2021. Available at: https://seer.cancer. $\mathrm{gov} /$ statfacts/html/lungb.html

27. Romito F, Dellino M, Loseto G, et al. Psychological distress in outpatients with lymphoma during the COVID-19 pandemic. Front Oncol 2020;10: 1270.

28. Han J, Zhou F, Zhang L, et al. Psychological symptoms of cancer survivors during the COVID-19 outbreak: a longitudinal study. Psychooncology 2021;30:378-384.

29. Higgins ET. Knowledge activation: accessibility, applicability, and salience. In: Higgins ET, Kruglanski AW, eds. Social Psychology: Handbook of Basic Principles. New York, NY: Guilford Press; 1996.

30. Walker J, Mulick A, Magill N, et al. Major depression and survival in people with cancer. Psychosom Med 2021;83:410-416.

31. Sprangers MA, Schwartz CE. Integrating response shift into healthrelated quality of life research: a theoretical model. Soc Sci Med 1999; 48:1507-1515.

32. Southwick SM, Bonanno GA, Masten AS, et al. Resilience definitions, theory, and challenges: interdisciplinary perspectives. Eur J Psychotraumatol 2014;5:1

33. Vella SLC, Pai NB. A theoretical review of psychological resilience: defining resilience and resilience research over the decades. Arch Med Health Sci 2019;7:233-239.

34. Brown Johnson CG, Brodsky JL, Cataldo JK. Lung cancer stigma, anxiety, depression, and quality of life. J Psychosoc Oncol 2014;32:59-73.

35. Panchal N, Kamal R, Orgera K, et al. The implications of COVID-19 for mental health and substance use. Accessed June 3, 2021. Available at: https://abtcounseling.com/wp-content/uploads/2020/09/The-Implicationsof-COVID-19-for-Mental-Health-and-Substance-Use-_-KFF.pdf 
36. Centers for Disease Control and Prevention. COVID data tracker. Accessed June 3, 2021. Available at: https://covid.cdc.gov/covid-datatracker/\#datatracker-home

37. Hyland KA, Jim HSL. Behavioral and psychosocial responses of people receiving treatment for advanced lung cancer during the COVID19 pandemic: a qualitative analysis. Psychooncology 2020;29: 1387-1392.
38. Abbasi S, Badheeb A. Prognostic factors in advanced non-small-cell lung cancer patients: patient characteristics and type of chemotherapy. Lung Cancer Int 2011;2011:152125.

39. Joshy G, Thandrayen J, Koczwara B, et al. Disability, psychological distress and quality of life in relation to cancer diagnosis and cancer type: population-based Australian study of 22,505 cancer survivors and 244,000 people without cancer. BMC Med 2020;18:372.

See JNCCN.org for supplemental online content.

NCCN

Pharmacy Updates

\section{Upcoming Live Webinars}

Not Just a Band-Aid: The Impact of Chemotherapy Dose Banding in Infants

Friday, February 25, 2022 • 1:30 - 2:30 PM EST

Alexis Kuhn, PharmD, BCOP, Mayo Clinic Cancer Center

\section{Crossroads of Exercise and Chemotherapy}

Thursday, March 10, 2022 • 12:00 - 1:00 PM EST

Brianna Archambeau, PharmD, BCOP, The Ohio State University Comprehensive Cancer Center -

James Cancer Hospital and Solove Research Institute

BCOP credit and continuing education credit/contact hours are available!

Visit education.nccn.org/pharmacy-updates to register for upcoming webinars,

access previous presentations, and view full accreditation information.

This activity is supported by educational grants from AstraZeneca; Bayer HealthCare Pharmaceuticals Inc.; Daiichi Sankyo; Epizyme Inc.; Novartis; and Sanofi Genzyme. This activity is supported through independent medical education grants from Merck \& Co., Inc., and Regeneron Pharmaceuticals, Inc and Sanofi Genzyme. This activity is supported by an education grant from Seagen Inc. formerly Seattle Genetics. 
Supplemental online content for:

\section{Cancer Treatment During COVID-19: Resilience of Individuals With Advanced Non-Small Cell Lung Cancer Versus Community Controls}

Nicole A. Arrato, MA; Stephen B. Lo, MA; Clarence A. Coker, BSc; Jonathan J. Covarrubias, MS; Tessa R. Blevins, BA; Sarah A. Reisinger, PhD, MPH, MCHES; Carolyn J. Presley, MD, MHS;

Peter G. Shields, MD; and Barbara L. Andersen, PhD

J Natl Compr Canc Netw 2022;20(2):118-125

eTable 1: Eligibility Criteria for NCT03199651 Cohort Study Enrollment

eTable 2: COVID-19 Assessment Items 


\section{eTable 1. Eligibility Criteria for NCT03199651 Cohort Study Enrollment}

\section{Inclusion criteria}

- Newly diagnosed, pathologically confirmed stage IV NSCLC

- Any performance status and any comorbidity

- Age $\geq 18$ y

- Treated at an Ohio institution within the network established for this study at the time of enrollment

- English-speaking

- Willingness to provide access to medical records, insurance, and billing data, biospecimens and to respond to questionnaires

\section{Exclusion criteria}

- Treatment with definitive chemotherapy/radiotherapy

- Receiving NSCLC treatment longer than 1 month before enrollment

- Presence of disabling hearing, vision, or severe psychiatric impairments preventing consent or completion of patient-reported outcomes

Abbreviation: NSCLC, non-small cell lung cancer.

\section{eTable 2. COVID-19 Assessment Items}

\begin{tabular}{|c|c|c|}
\hline Item & Description & Range \\
\hline \multicolumn{3}{|l|}{ COVID-19 illness perceptions } \\
\hline Consequences & $\begin{array}{l}\text { How much is the spread of COVID-19 in the } \\
\text { United States affecting your life? }\end{array}$ & 0 (no effect at all) to 10 (severely affects my life) \\
\hline Anticipated timeline & $\begin{array}{l}\text { How long do you think the COVID-19 pandemic } \\
\text { will continue? }\end{array}$ & 0 (a very short time) to 10 (forever) \\
\hline Personal control & $\begin{array}{l}\text { How much control do you feel you have over } \\
\text { getting COVID-19? }\end{array}$ & 0 (absolutely no control) to 10 (extreme amount of control) \\
\hline Comprehension & How well do you understand what COVID-19 is? & $\begin{array}{l}0 \text { (do not understand it at all) to } 10 \text { (I understand } \\
\text { it very clearly) }\end{array}$ \\
\hline Concern (COVID-19) & How concerned are you about getting COVID-19? & 0 (not at all concerned) to 10 (extremely concerned) \\
\hline Concern (cancer/health) & $\begin{array}{l}\text { Has the COVID-19 pandemic worsened your } \\
\text { concern about your lung cancer ("health" in } \\
\text { healthy survey)? }\end{array}$ & 0 (not at all concerned) to 10 (extremely concerned) \\
\hline \multicolumn{3}{|l|}{ COVID-19 infection tally } \\
\hline Family & How many family members have been diagnosed? & $0(0)-10(\geq 10)$ \\
\hline Friend & How many friends? & $0(0)-10(\geq 10)$ \\
\hline Acquaintance & $\begin{array}{l}\text { How many others do you know that have been } \\
\text { diagnosed with COVID-19, such as coworkers? }\end{array}$ & $0(0)-10(\geq 10)$ \\
\hline \multicolumn{3}{|l|}{ Social distancing } \\
\hline Within household & $\begin{array}{l}\text { In the past } 7 \text { days in your household, have you } \\
\text { been able to socially distance yourself from } \\
\text { household members? }\end{array}$ & 0 (not at all) to 6 (yes, all the time) \\
\hline \multicolumn{3}{|l|}{ COVID-19 stress } \\
\hline Personal stress & $\begin{array}{l}\text { In the past } 7 \text { days, how would you rate your } \\
\text { stress during isolation or social distancing? }\end{array}$ & 0 (no stress at all) to 6 (extreme amount of stress) \\
\hline Household stress & $\begin{array}{l}\text { In the past } 7 \text { days, has isolation or social } \\
\text { distancing created tension in your household? }\end{array}$ & 0 (no tension at all) to 6 (extreme amount of tension) \\
\hline Family worry & $\begin{array}{l}\text { How worried are you that any of your close family } \\
\text { members will contract COVID-19? }\end{array}$ & 0 (not worried at all) to 10 (extremely worried) \\
\hline
\end{tabular}

